\title{
Minimum Radiation Q for Spheroids - Extension to Cylinder, Comparison to Spherical Formulas and Practical Antennas
}

\author{
AP-S International Symposium \\ Peder M. Hansen*, and Richard Adams \\ SPAWAR Systems Center Pacific - Code 554 \\ Electromagnetics and Advanced Technology Division \\ San Diego, CA 92152 \\ E-mail: peder.hansen@navy.mil
}

\section{Introduction}

The radiation $\mathrm{Q}\left(Q_{\mathrm{r}}\right)$ of an antenna is defined to be the antenna $\mathrm{Q}$ when the only loss is radiated power. It is well known that electrically small antennas have large radiation $Q$. Large $Q_{r}$ results in narrow bandwidth, reduced efficiency due to losses in tuning elements and high near fields, as well as high voltages and currents that limit the amount of radiated power. For each of these considerations it is important to minimize $\mathrm{Q}_{\mathrm{r}}$ of an ESA.

There are fundamental limits on the radiation Q of an ESA that depend upon the size and shape of the volume containing the antenna. Considerable effort has been expended over the years in an attempt to understand and quantify these limits $[1,2]$. Much of these efforts involved determining the minimum $\mathrm{Q}_{\mathrm{r}}$ that can be achieved by an antenna contained in a spherical volume with no energy stored inside [3, 4]. For this case, exact analytic formulas for the minimum possible radiation $Q$ can be obtained when the sphere is electrically small. Unfortunately, the assumption of no energy stored within the sphere is not realistic and the resulting bound is well below the results for practical antennas. Some recent efforts have been directed towards including energy stored within the sphere $[5,6]$. Figure 1 gives curves comparing results from various formulas for the spherical case, including exact formulas developed by McLean and Grimes [3, 4] and results by Hansen and Collin that include internal energy [6].

\section{Approach}

The analysis for the sphere has been extended to a spheroid in an attempt to understand the effect of shape as described by the aspect ratio. The approach used was to expand the fields in spheroidal modes and integrate the stored energy and radiated power following McLean. Analytic solutions are not possible for this case but numerical results have been obtained [7,8]. For the spherical case, the minimum $\mathrm{Q}_{\mathrm{r}}$ results when only the lowest order (dipole) mode is present. For the spheroidal case the minimum $Q_{r}$ requires the sum of the first two modes.

Results for the electrically small case are given in Figure 2 for $\mathrm{Q}_{\mathrm{r}}$ of the lowest order mode and the minimum $\mathrm{Q}_{\mathrm{r}}$ for the two mode sum, normalized to the 
minimum $\mathrm{Q}_{\mathrm{r}}$ for a sphere with the same height. For aspect ratios well away from 1 the minimum $\mathrm{Q}_{\mathrm{r}}$ of the two mode sum is orders of magnitude lower than that for the lowest order mode alone. When the aspect ratio is near 1, a sphere, the two curves converge and the lowest order mode gives the minimum $\mathrm{Q}_{\mathrm{r}}$.

Figure 2 shows two separate analytic fits to the minimum $\mathrm{Q}_{\mathrm{r}}$ curve, one for oblate $(\mathrm{b} / \mathrm{a} \leq 1)$ and the other for prolate $(\mathrm{b} / \mathrm{a}>1)$. The fit for oblate is given by equation 1 with the coefficients in Table 1, where (ar) is the aspect ratio (b/a).

$$
\min Q_{r} / Q_{s p h}=\frac{A+B \cdot \ln (a r)+C \cdot \ln ^{2}(a r)+D \cdot \ln ^{3}(a r)}{1+E \cdot \ln (a r)+F \cdot \ln ^{2}(a r)+G \cdot \ln ^{3}(a r)+H \cdot \ln ^{4}(a r)} \quad \mathrm{b} / \mathrm{a} \leq 1
$$

\begin{tabular}{|c|c|c|c|}
\hline \multicolumn{4}{|c|}{ Table 1 Numerical Coefficients for Equation 8 } \\
\hline $\mathrm{A}$ & $\mathrm{B}$ & $\mathrm{C}$ & $\mathrm{D}$ \\
\hline 0.9962826 & 0.694527 & 0.1638087 & 0.0129323 \\
\hline $\mathrm{E}$ & $\mathrm{F}$ & $\mathrm{G}$ & $\mathrm{H}$ \\
\hline-0.8297389 & 1.0034151 & 0.2218167 & 0.50818055 \\
\hline
\end{tabular}

Equation 2 is the fit for prolate $(b / a \geq 1)$ with the coefficients in Table 2.

$$
\min Q_{r} / Q_{s p h}=A+B \cdot(a r)+C \cdot \ln ^{2}(a r)+D \cdot \sqrt{a r}+E / \sqrt{a r} \quad \mathrm{~b} / \mathrm{a} \geq 1
$$

\begin{tabular}{|c|c|c|c|c|}
\hline \multicolumn{5}{|c|}{ Table 2 Numerical Coefficients for Equation 9 } \\
\hline A & B & C & D & E \\
\hline 63.873474 & 1.0814504 & 8.2821637 & -31.419134 & -32.53629 \\
\hline
\end{tabular}

These equations can be used to determine the minimum $Q_{\mathrm{r}} / Q_{\mathrm{sph}}$ for a spheroidal shape over wide range of aspect ratios. For both formulas the normalization factor is $(1 / \mathrm{kb})^{3}$, where $b$ is the radius of a sphere the same height as the spheroid.

\section{Application to Cylinder}

The formulas have been used to develop a bound for a cylinder for a range of cylinder aspect ratios and the results shown in Figure 3 "lower bound". Figure 3 also has curves for various antennas that fit within the cylinder. These curves give a direct comparison of antennas having the same height and footprint. The results shown were calculated for an antenna height of 0.3 meters or $0.01 \lambda$ at 10 $\mathrm{MHz}$ and are all normalized to the minimum $\mathrm{Q}_{\mathrm{r}}$ for a sphere of the same height. The general shape of the curves for practical antennas is similar to the bound for the cylinder, although starting to diverge for small aspect ratios. Over much of the range, $Q_{r}$ for the practical antennas is $2-3$ times the cylinder bound. There are two reasons for this, first the analysis does not include internal stored energy and second the spheroid used to obtain the bound is larger than the cylinder.

\section{References}


[1] Hansen, R.C., "Electrically Small Superdirective and Superconducting Antennas", Wiley-Interscience, Hoboken, NJ, 2006, Chapter 1 references [2] Slyusar, V.I., "60 Years of Electrically Small Antennas", "IEEE International Conference on Antenna Theory and Techniques, 17-21 September, 2007 Sevastopol, Ukraine, March 1978, pp116-118. http://www.slyusar.kiev.ua/Slyusar_077.pdf [3] McLean, "A Re-examination of the Fundamental Limits on the Radiation Q of Electrically Small Antennas" IEEE Transactions on Antennas and Propagation, AP-44, May 1996, pp 672-675

[4] D. M. Grimes and C. A. Grimes, "Radiation Q of dipole generated fields," Radio Science, 34, pp. 282-296, 1999.

[5] Thal, Herbert L. Jr., "New Radiation Q Limits for Spherical Wire Antennas", IEEE TAP, Vol. 54, No. 10, October 2006.

[6] Hansen, R.C. and R.E. Collin, "Chu Explicated", Submitted to IEEE Antennas and Propagation Magazine.

[7] Adams, R.C. and P.M. Hansen, "The Evaluation of 'Q' in an Electrically Small Antenna in Prolate Spheroidal Coordinates", Technical Document 3188, published September 2004, located at the web site:

http://www.spawar.navy.mil/sti/publications/pubs/tdsbynumber.html

[8] Adams, R.C. and P. M. Hansen, "The Modal Decomposition of the Quality Factor of an Antenna in Prolate Spheroidal Coordinates", Technical Report 1969, published September 2008, located at the web site:

http://www.spawar.navy.mil/sti/publications/pubs/trindex.html

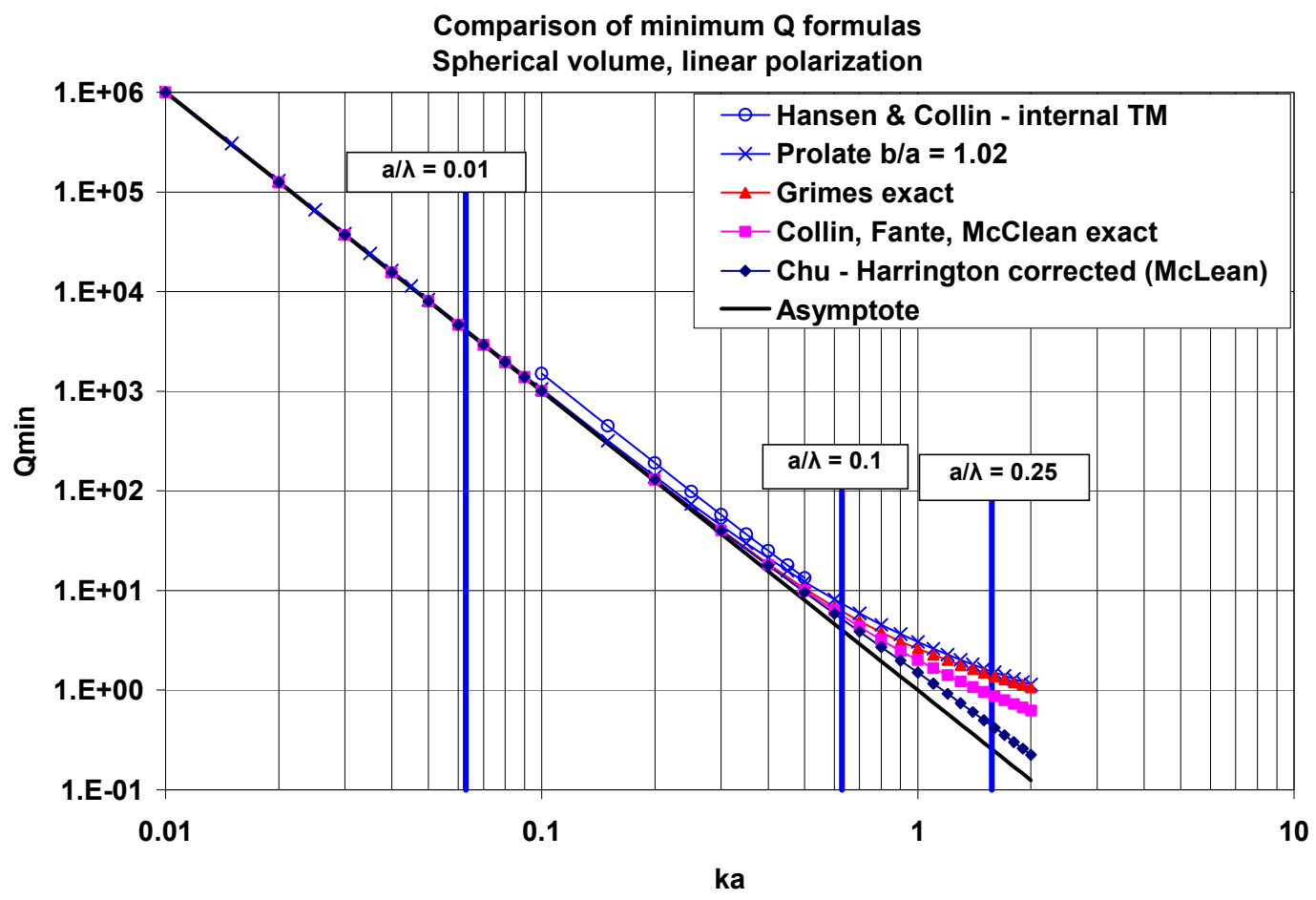

Figure 1 Comparison of minimum $\mathrm{Q}_{\mathrm{r}}$ formulas for sphere. 


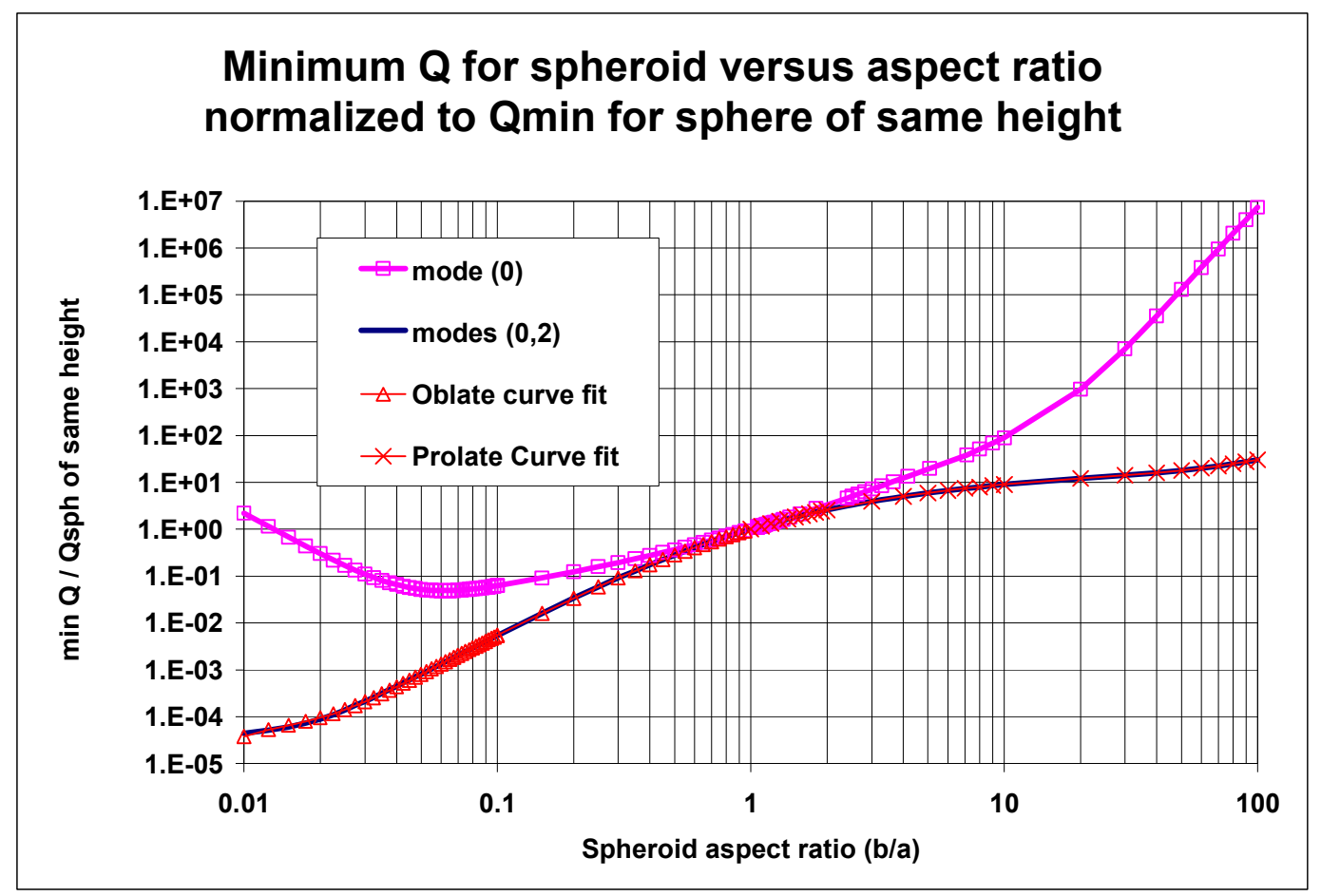

Figure 2 Normalized minimum $\mathrm{Q}_{\mathrm{r}}$ for antenna contained within a spheroid.

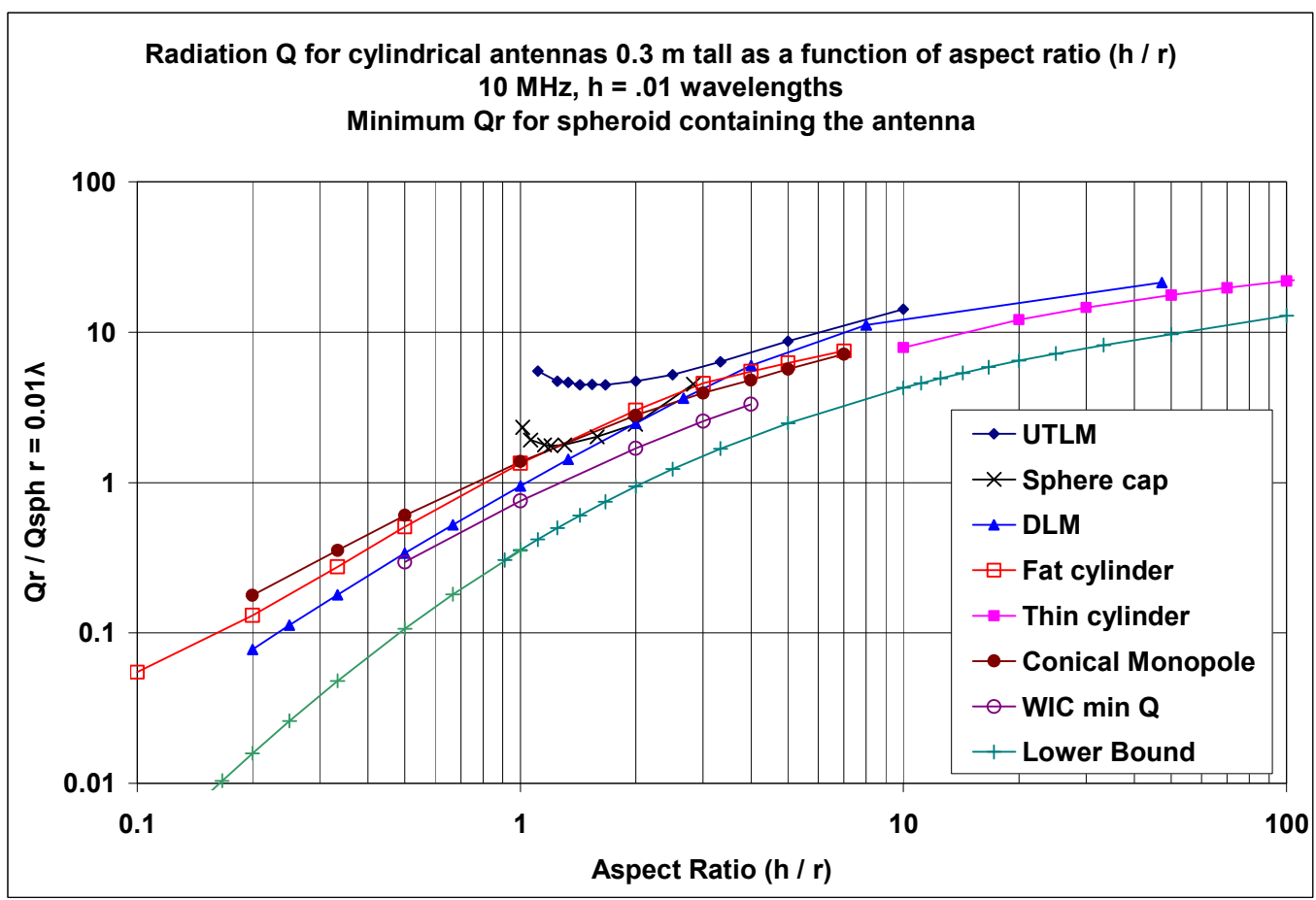

Figure 3 Comparison of normalized $\mathrm{Q}_{\mathrm{r}}$ for various practical antennas and lower bound for a cylinder. 